\title{
AUTOMEDICAÇÃO: ATUAÇÃO DO FARMACÊUTICO NA PREVENÇÃO A INTOXICAÇÃO MEDICAMENTOSA
}

\author{
SELF-MEDICATION: PHARMACIST'S PERFORMANCE IN THE PREVENTION \\ OF DRUG INTOXICATION
}

\author{
Renata Carvalho de Oliveira Souza ${ }^{1}$ \\ Leonardo Guimarães de Andrade ${ }^{2}$
}

\begin{abstract}
RESUMO: A intoxicação medicamentosa é compreendida como uma sequência de manifestações e sintomas motivados pela ingestão incorreta de um medicamento, esse fato é capaz de alterar o nosso organismo, e causar sintomas indesejáveis. O farmacêutico é o profissional mais apto a falar sobre o uso racional de medicamentos e tratar da intoxicação medicamentosa pois é conhecedor técnico quanto ao assunto. Com base nisso objetiva-se garantir a importância do profissional farmacêutico na prevenção a intoxicação medicamentosa. Utilizou-se o método de revisão bibliográfica com dados extraídos de várias plataformas de dados científicos, além de documentos oficiais dos órgãos responsáveis pela sistematização pelos dados relacionados à saúde.
\end{abstract}

Palavras-chave: Automedicação. Atuação farmacêutica. Intoxicação medicamentosa. Uso racional de medicamentos.

ABSTRACT: Drug intoxication is understood as a sequence of manifestations and symptoms motivated by the incorrect ingestion of a drug, this fact is capable of altering our organism, and causing undesirable symptoms. The pharmacist is the most qualified professional to talk about the rational use of medicines and deal with drug intoxication because he is technically knowledgeable about the subject. Based on this, the objective is to guarantee the importance of the pharmacist in preventing drug intoxication. The literature review method was used with data extracted from various scientific data platforms, in addition to official documents from the bodies responsible for systematizing health-related data.

Keywords: Self medication. Pharmaceutical performance. Drug poisoning. Rational use of medicines.

\footnotetext{
I Em técnico de Administração em 2002. Graduação em processo de finalização em farmácia. Graduanda na Universidade Iguaçu. Nova Iguaçu, Estado do Rio Janeiro.

${ }^{2}$ Orientador. UNIG - Universidade Iguaçu.
} 


\section{INTRODUÇÃO}

Desde os tempos antigos, sempre houve a preocupação em curar doenças e trazer alívio aos indivíduos acometidos por enfermidades físicas ou emocionais. As civilizações primárias já fabricavam óleos e unguentos provenientes de plantas medicinais, utilizados por xamãs e raizeiros, hoje essa ciência que estuda o tratamento através das plantas é denominada fitoterapia (NOGUEIRA, 2009).

Muitas vezes ao buscarem tratamentos milagrosos e trazer alívio a enfermidades, pessoas manipulavam substâncias e acabaram morrendo intoxicadas por não conhecerem a toxicidade, dosagem e formas de administração corretas (MAGALHÃES, 2016).

A utilização incorreta de medicamentos, é capaz de alterar o nosso organismo, e causar sintomas indesejáveis, a intoxicação medicamentosa possui várias causas (uso incorreto, dosagem erradas, prescrição incorreta etc.), se tornou um problema de saúde pública em todo o mundo, ocasionando internamentos e mortes (SCHENKEL, 2004).

Alguns autores citam que a ingestão irregular de medicamentos são os mais responsáveis pelas causas de intoxicação, fato este que é relacionado com o apelo das publicidades irregulares, facilidade de compra e deficiência dos programas educativos em saúde, o que leva ao uso indiscriminado (MOTA et al., 2012)

Objetiva-se nesse trabalho destacar a importância do profissional farmacêutico na orientação do uso seguro e racional de medicamentos, dando destaque sobre intoxicação farmacológica, retratando índices de intoxicação e ações da assistência farmacêutica no controle da intoxicação.

\section{OBJETIVOS}

\subsection{Objetivo geral}

Avaliar a atuação do profissional farmacêutico, ao implementar a ferramenta da atenção farmacêutica com intuito de reduzir os riscos e danos provocados pela intoxicação medicamentosa.

\subsection{Objetivos Específicos}

- Alertar sobre o risco da prática da automedicação; 
- Abordar sobre o uso racional de medicamentos;

- Analisar os fatores que pré-dispõem e os sintomas causados pela intoxicação medicamentosa;

- Identificar as principais intoxicações medicamentosas;

- Assegurar a importância do profissional farmacêutico na prevenção da intoxicação medicamentosa por meio de ações preventivas.

\section{METODOLOGIA}

Trata-se de estudo exploratório realizado por meio de revisão narrativa da literatura científica, utilizando materiais coletados por meio das bases de dados: SciELO - Scientific Eletronic Library, Google Acadêmico, periódicos, monografias, congressos, legislações, revistas científicas e Ministério da Saúde (MS) que datam do ano de 2004 a 202I.Como critério de inclusão foram pré-definidos os materiais de idiomas português, inglês e espanhol, artigos que dissertavam sobre atuação farmacêutica, uso indevido de medicamentos e intoxicação medicamentosa. Os critérios de exclusão utilizados foram os artigos com temas que não corroboravam com o tema, fora do período escolhido e os de outras línguas que não foram mencionadas anteriormente.

\section{JUSTIFICATIVA}

O consumo excessivo de medicamento, a automedicação e o fácil acesso da população são considerados um fator perigoso e prejudicial à saúde pública, sendo classificada como a principal causa de intoxicação no país. Segundo o Sistema Nacional de Informações Tóxico-Farmacológicas (SINITOX) este é apontado como o primeiro lugar por intoxicação desde o ano de 1994, tendo como as principais classes causadoras de intoxicação os antigripais, antidepressivos, anti-inflamatórios e benzodiazepínicos (FERREIRA, 2014)

Justifica-se nesse trabalho versar por meio de uma revisão bibliográfica, a atuação do profissional farmacêutico na prevenção da intoxicação medicamentosa, com intuito de que esse material seja útil para consultas, objetivando a conscientização da população em relação aos riscos causados por essa prática. 


\section{REVISÃO DE LITERATURA}

\section{I Automedicação como fator determinante para intoxicação medicamentosa}

A automedicação é designada como a ingestão de medicamentos sem prescrição ou sem orientação de um profissional de saúde capacitado para tal. Para Kovacs e Brito (2006) a automedicação é definida como "uso de medicamentos sem prescrição médica, onde o próprio paciente decide qual droga vai utilizar, podendo ser utilizado remédios industrializados ou caseiros".

Já a intoxicação medicamentosa é compreendida como uma sequência de manifestações e sintomas motivados pela ingestão de um medicamento ou quando o fármaco é injetado, inalado, ou entra em contato com a mucosa dos olhos, pele ou membranas em doses exacerbadas, acima da terapêutica prescrita para cada indivíduo. Problemas de intoxicação medicamentosa podem ser contornados através de ações educativas que visam promover saúde, tendo por objetivo o uso racional de medicamentos, destacando a atuação do farmacêutico integrando as equipes multidisciplinares (GONÇALVES et al., 2017).

As intoxicações medicamentosas podem ser agudas ou crônicas, a característica que as diferencia são que as agudas possuem reações que vão de vinte e quatro horas a duas semanas, e as crônicas de três meses a anos de exposição ao agente tóxico (OLSON et al., 2013).

A automedicação é um problema multicausal, estimulada pela facilidade de adquirir medicamentos, propagandas de marketing que impulsionam as pessoas a comprarem medicamentos sem necessidade, por indicações de amigos e familiares, pelo fácil acesso através de compras na internet, porém, essa prática traz consequências desagradáveis, causadas pelo uso irregular de medicamentos (DHAMER et al., 2012).

O uso inadequado de medicamentos é um problema que traz um custo ao serviço de saúde, o Brasil é $5^{o}$ país no mundo com maior consumo de medicamentos, e o I $^{\mathrm{O}}$ na América Latina (SOUSA et al., 2008). Um estudo conduzido por Lima et al., (2008) relata que qualquer substância pode causar intoxicação. Já Paracelsus dizia: “o que diferencia a dose correta para o veneno é a dose ingerida" (NOGUEIRA, 2009).

De acordo com o Instituto de Pesquisa e Pós-Graduação para o mercado farmacêutico (ICTQ) os medicamentos mais consumidos no Brasil são: analgésicos (48\%), 
anti-inflamatórios (20\%), antialérgicos (13\%), antibióticos (8\%) e ansiolíticos (tarja preta) (3\%) (ICTQ, 2021).

Aquino (2008) relata que as intoxicações medicamentosas causam inestimável perda de vidas, sobretudo das pessoas mais vulneráveis economicamente, as quais utilizam de um serviço público já debilitado, pessoas com menor índice de escolaridade, menor poder aquisitivo e crianças são as mais prejudicadas em decorrência da falta de orientação.

Embora não demonstrem a quantidade real de casos devido as subnotificações, dados do Sistema Nacional de Informações Tóxico-Farmacológicas (SINITOX) corroboram com as informações sobre a influência da automedicação como fator predominante na intoxicação medicamentosa (TABELA I).

TABELA I: Casos e Mortes causadas por Intoxicação Medicamentosa no Brasil

\begin{tabular}{|c|c|c|c|c|c|c|c|}
\hline Região & & 2013 & 2014 & 2015 & 2016 & 2017 & Total \\
\hline Norte & $\begin{array}{l}\text { Casos } \\
\text { Mortes }\end{array}$ & $\begin{array}{l}383 \\
I\end{array}$ & $\begin{array}{l}202 \\
-\end{array}$ & $\begin{array}{l}263 \\
-\end{array}$ & $\begin{array}{l}- \\
-\end{array}$ & $\begin{array}{l}394 \\
-\end{array}$ & $\begin{array}{l}\mathrm{I} 242 \\
\mathrm{I}\end{array}$ \\
\hline Nordeste & $\begin{array}{l}\text { Casos } \\
\text { Mortes }\end{array}$ & $\begin{array}{l}1804 \\
9\end{array}$ & $\begin{array}{l}1736 \\
8\end{array}$ & $\begin{array}{l}1939 \\
22\end{array}$ & $\begin{array}{l}2459 \\
14\end{array}$ & $\begin{array}{l}640 \\
2\end{array}$ & $\begin{array}{l}8578 \\
55\end{array}$ \\
\hline SUDESTE & $\begin{array}{l}\text { Casos } \\
\text { Mortes }\end{array}$ & $\begin{array}{l}7778 \\
35\end{array}$ & $\begin{array}{l}13496 \\
25\end{array}$ & $\begin{array}{l}15826 \\
15\end{array}$ & $\begin{array}{l}18731 \\
72\end{array}$ & $\begin{array}{l}7658 \\
24\end{array}$ & $\begin{array}{l}63489 \\
171\end{array}$ \\
\hline $\begin{array}{l}\text { CENTRO } \\
\text { OESTE }\end{array}$ & $\begin{array}{l}\text { Casos } \\
\text { Mortes }\end{array}$ & $\begin{array}{l}4011 \\
12\end{array}$ & $\begin{array}{l}1973 \\
7\end{array}$ & $\begin{array}{l}1353 \\
3\end{array}$ & $\begin{array}{l}979 \\
4\end{array}$ & $\begin{array}{l}425 \\
I\end{array}$ & $\begin{array}{l}874 \mathrm{I} \\
27\end{array}$ \\
\hline BRASIL & $\begin{array}{l}\text { Casos } \\
\text { Mortes }\end{array}$ & $\begin{array}{l}23549 \\
78\end{array}$ & $\begin{array}{l}26593 \\
6 \mathrm{I}\end{array}$ & $\begin{array}{l}28778 \\
62\end{array}$ & $\begin{array}{l}32311 \\
\text { II5 }\end{array}$ & $\begin{array}{l}20637 \\
50\end{array}$ & $\begin{array}{l}131868 \\
366\end{array}$ \\
\hline
\end{tabular}

FONTE: SINITOX, 2020

Estima-se que esses dados representem apenas 20\% dos casos que ocorrem anualmente, de toda forma esses registros obtidos pelos centros de informação e assistência toxicológica norteiam a atuação da Vigilância Sanitária e da Segurança Pública (PAPINI, NAKAGAWA, 2014).

\subsection{Uso racional de medicamentos}

O uso racional de medicamentos (URM) é tido como um dos elementos-chave proposto pela Organização Mundial de Saúde (OMS) para as políticas de medicamentos (BRASIL, 2012). Na Política Nacional de Medicamentos (PNM) do Brasil, ele é definido como: "o processo que compreende a prescrição apropriada; a disponibilidade oportuna e a 
preços acessíveis; a dispensação em condições adequadas; e o consumo nas doses indicadas, nos intervalos definidos e no período de tempo indicado de medicamentos eficazes, seguros e de qualidade" (BRASIL, 2012).

Para implementar o URM é primordial que se desenvolva estratégias como a seleção de medicamentos, farmacovigilância, dispensação e uso apropriado de medicamentos, gerenciamento adequado dos serviços farmacêuticos, educação dos usuários quanto aos riscos da automedicação construção de formulários terapêuticos, da interrupção e da troca de medicamentos prescritos (ESHER; COUTINHO, 2017).

\subsection{Fatores que predispõem a intoxicação medicamentosa}

Uma das preocupações impostas aos profissionais que trabalham com fármacos desde a sua fabricação, manipulação, distribuição, prescrição e dosagem, é a de que tratamos com vidas em todas os estágios desse processo, uma vez que, falhas e acertos advindos desses profissionais podem trazer consequências irreversíveis como a morte, ou ainda sequelas e intoxicações aos pacientes e a eles próprios (OLIVEIRA, 2009).

Mesmo sendo elaborados para auxiliar no tratamento e controle de doenças, os medicamentos são campeões quando se trata de intoxicação, seja quando o indivíduo atenta contra a própria vida, quando há erros na administração, quando ocorrem superdosagens ou são tomados acidentalmente, o uso incorreto dessas drogas chega a ser responsável por até $30 \%$ das causas de intoxicação no Brasil, taxa maior do que as intoxicações por metais, produtos de limpeza e agrotóxicos (VARGAS, 2019).

As intoxicações são resultantes das manifestações do fármaco no organismo, que podem causar variações químicas e fisiológicas, a qual se origina a ação patológica, mediada por substâncias químicas endógenas ou exógenas, a partir daí surgem sinais e sintomas, geralmente causadas por altas dosagens (OGA et al., 2014).

Os principais sintomas causados pelo uso de medicamentos sem prescrição médica são: tosse seca, congestão nasal, broncoespasmo, febre, cefaleia, diarreia, má digestão e dores abdominais (CRUZ et al., 2014).

O consumo de fármacos é estimulado por propagandas de grandes empresas, e faz parte de uma economia que só cresce. Segundo uma consultoria norte-americana (IMS Health), especializada na área farmacêutica, o mercado brasileiro de farmácia atingiu $\mathrm{R} \$ 38$ 
bilhões de reais em vendas só no ano de 20II, no ano anterior foram vendidos mais de 2 milhões de unidades de remédios, a questão é que inúmeros medicamentos comprados são resultantes da automedicação, do uso incorreto e por vezes desnecessário, o que expõe as pessoas a um risco maior de reações adversas e intoxicações (MAIOR E OLIVEIRA, 2012).

Informações coletadas pelo Centro de Assistência Toxicológica (CEATOX) no decorrer de uma década (I991 a 2000), apurou um acréscimo na incidência de intoxicação medicamentosa dentre eles pode-se ressaltar: Gardenal (fenobarbital), Valium (Diazepam), Haldol (haloperidol), tegretol (Carbamazepina), lexotan (Bromazepam) (SÃO PAULO, 2014). As taxas de intoxicação das seguintes classes medicamentosas são: benzodiazepínicos (14,8\%), anticonvulsivantes (9,6\%), antidepressivos (6,9\%) e analgésicos $(6,5 \%)$, desses em $85 \%$ dos casos não houve mortes (BITENCOURT et al., 2008).

As três principais circunstâncias que levam a intoxicação medicamentosa são, tentativa de suicídio 38,1\% (50.392), ingestão acidental 31,64\% (4I.723) e o uso terapêutico I2,57\% (16.58I). Geralmente quando o indivíduo tenta contra a própria vida, utiliza mais de uma substância medicamentosa ou não, em relação a esse tipo de causa as drogas psicotrópicas, principalmente sedativos, anticonvulsivantes e antidepressivos, são as mais utilizadas (BERNARDES, TURINI, MATSUO, 2ого).

Os fatores que mais predispõem os indivíduos a intoxicação medicamentosa são: o ato de tomar medicamento sem orientação profissional, erro de prescrição (receitas médicas inelegíveis), exposição acidental (crianças tendo acesso a medicamentos armazenados em locais de fácil acesso), utilização inadequada (em dose maior ou por mais tempo do que a prescrita pelo profissional que prescreveu) e dentre esses fatores os que podemos destacar; tentativas de suicídio e aborto, esses dois últimos mais comuns entre mulheres mais jovens (GANDOLFI, 2006).

A maioria dos casos de intoxicação medicamentosa ocorre nas áreas urbanas 85,71\% (II3.030), pela facilidade de comprar medicamentos, também justificada pela existência das "farmacinhas caseiras" que por sua vez, aumentam o risco da ingestão indevida, armazenamento inadequado e inobservância da data de validade (MAIOR; OLIVEIRA, 2012). 
As mulheres são as maiores vítimas de intoxicação medicamentosa (54\%) e morte, fato este que se dá motivado pela automedicação, segundo estudos essas mulheres são portadoras de doenças crônicas e mais atentas aos sinais de dor. Já os homens tendem a negar qualquer tipo de debilidade (PONS et al., 2017; ARRAIS et al., 2016).

As crianças fazem parte de uma parcela importante nas causas de intoxicação, a faixa de i a 4 anos tem as maiores taxas (27,07\% - 35.706), pela curiosidade comum da idade, é comum que coloquem tudo na boca, o que predispõe a uma exposição maior aos medicamentos e substâncias nocivas (MAIOR e OLIVEIRA, 2012).

Devido aos problemas emocionais, os adolescentes têm sido cada vez mais vítimas da intoxicação, tentados a comer o suicídio eles recorrem ao abuso na tomada de medicamentos. Salienta-se que a automedicação, a tomada excessiva de medicamentos junto ao desconhecimento da dose recomendada são fatores que levam crianças e adolescentes a cursarem com intoxicação medicamentosa, os adolescentes com problemas psicológicos e emocionais são induzidos a tentarem suicídio tomando uma grande quantidade de fármacos (SOUSA, 2021).

Acometidos por doenças crônicas e debilidades típicas da senilidade, os idosos tendem a tomar mais medicamentos e em decorrência estão mais sujeitos a reações adversas e intoxicações. $O$ ato de envelhecer induz o organismo uma maior vulnerabilidade, motivados por mudanças bioquímicas, fisiológicas e psicológicas, associado a uma tomada de vários medicamentos, essa faixa etária tem um risco maior às intoxicações medicamentosas (GONÇALVES et al., 2019).

\subsection{Causas, sintomas e tratamentos da intoxicação medicamentosa}

A maior parte das intoxicações é decorrente do desconhecimento do efeito nocivo das substâncias, seja estimulado pelas propagandas ou por não compreenderem a complexidade dos compostos ativos, é evidente que as causas acabam sendo a tomada inconsequente e exagerada, atrelada a falta de conhecimento sobre a dose, forma de administração, automedicação e a prescrição incorreta (GONÇALVES et al., 2019)

Um fator agravante para a intoxicação é a facilidade de adquirir tais medicamentos seja em farmácias ou pela internet, as embalagens coloridas e chamativas, fazem o consumidor ser induzido a comprar e armazenar de forma inadequada nas residências. A 
classe dos analgésicos, descongestionantes nasais, broncodilatadores, e os contraceptivos orais são os fármacos mais comprados e os que mais podem causar intoxicações (SOUSA et al., 2021).

Quando a tomada de medicamentos é voluntária, objetivando causar um efeito adverso e intoxicação, é comum que sejam tomados vários tipos de medicamentos, o que demonstra que parte da população reconhece que não se deve misturar drogas e substâncias, como por exemplo psicotrópicas, sobretudo os sedativos, anticonvulsivantes e antidepressivos, essa classe são os mais comuns de serem usados em tentativas de suicídio (SERENO et al., 2020).

$\mathrm{Na}$ tabela abaixo serão listadas as causas mais comuns de intoxicação e óbitos no Brasil (TABELA 2).

TABELA 2: Casos e óbitos em decorrência de intoxicação medicamentosa no Brasil

\begin{tabular}{|c|c|c|c|c|c|c|}
\hline Circunstância & 2013 & 2014 & 2015 & 2016 & 2017 & Total \\
\hline Acidente Individual & 8357 & 9559 & 9090 & 9666 & 5051 & 41723 \\
\hline Acidente Coletivo & 56 & 70 & 79 & 52 & 17 & 274 \\
\hline Acidente Ambiental & 8 & 2 & I & 2 & - & 13 \\
\hline Acidente Ocupacional & 66 & 42 & 26 & 22 & 8 & 164 \\
\hline Uso Terapêutico & 1336 & 2534 & 5819 & 5939 & 953 & 16581 \\
\hline Presc. Médica Inadequada & 153 & 134 & 53 & $5 \mathrm{I}$ & 19 & 410 \\
\hline Erro de administração & 1372 & 2719 & 1249 & 1612 & 1392 & 8344 \\
\hline Automedicação & 542 & $78 \mathrm{I}$ & 998 & 1013 & 397 & 3731 \\
\hline Abstinência & 28 & I & 8 & 4 & 4 & 45 \\
\hline Tentativa de Suicídio & 10386 & 9202 & 984I & I0980 & 9983 & 50392 \\
\hline Tentativa de Aborto & 19 & 28 & 40 & 29 & I6 & 132 \\
\hline Uso indevido & 237 & 352 & 320 & 272 & Io6 & 1287 \\
\hline
\end{tabular}

FONTE: Extraído e adaptado de Sereno et al., 2020

Conforme os dados publicados, fica evidente que as causas mais comuns de morte em decorrência da intoxicação medicamentosa são pelas tentativas de suicídio (38,21\%), acidente individual (31,64\%) e o uso terapêutico (I2,57\%) (SERENO et al., 2020).

Ao apresentar indícios de intoxicação medicamentosa é imprescindível que os sinais vitais do paciente sejam checados: pressão arterial, pupilas, frequência cardíaca e respiratória, oxigenação e temperatura. Também deve-se averiguar vias aéreas, circulação sanguínea e déficit neurológico. Nos pacientes desacordados deve-se administrar glicose e 
tiamina (vitamina $\mathrm{BI}$ ), evitando que o paciente tenha uma hipoglicemia e possível diminuição de consciência (CASTILHO et al., 2018; PÉREZ et al., 2019; SILVA et al., 202ı).

Nem sempre há um antídoto para as intoxicações, Skolnik e Monas (2017) certificam de forma categórica que após estabilizar o paciente, deve-se encontrar o tratamento mais adequado levando em conta os antecedentes pessoais do mesmo.

Se a substância foi ingerida em um período de até quatro horas, apesar de não haver consenso médico, habitualmente é feita uma lavagem gástrica, utilizando carvão ativado, assim evitando que o organismo absorva totalmente o agente intoxicante (SÃO PAULO, 2017). Em contraposição ao consenso médico, o carvão ativado reduz consideravelmente os efeitos hepatotóxicos causados pela intoxicação por paracetamol (BEZERRA et al., 2020).

Nos pacientes que ingeriram superdoses de ácido acetilsalicílico e de barbitúricos é feita a utilização de bicarbonato de sódio intravenoso, que tem por consequência o aumento da excreção urinária dos fármacos, quando esse tratamento não é suficiente, o paciente é submetido a diálise peritoneal, a hemodiálise ou a hipoperfusão, com a finalidade de desintoxicar o sangue do agente intoxicante, esse procedimento é utilizado nas intoxicações por salicilatos, lítio e fenobarbital (FERRANTI et al., 20r8; SILVA et al., 202I).

Nas intoxicações por compostos lipofílicos, como antipsicóticos, antidepressivos tricíclicos e betabloqueadores, são utilizados de forma experimental emulsões lipídicas intravenosas, acredita-se que ao chegar no sangue esses compostos se unam a emulsão, se afastando dos receptores teciduais. A utilização de bicarbonato de sódio, objetivando a abertura dos canais de sódio prejudicados pelo excesso de antidepressivos tricíclicos e antipsicóticos é usualmente realizada na prática clínica (MARTINS; FILHO, 2018).

Anti-histamínicos como a ciproheptadina, também são utilizados nos tratamentos de intoxicações que causam síndrome serotoninérgicas, essa síndrome causa agitação, alucinações, midríase e em alguns casos, convulsões, comum na ingestão de fentanil, tramadol e antidepressivos tricíclicos (BARTLETT, 2017)

Os sintomas podem variar de acordo com o agente causador, podendo ter efeito sútil ou grave, que pode ser afetado pela dose consumida, inalada, injetada, no quadro abaixo veremos os principais agentes tóxicos e suas manifestações clínicas, além do tratamento utilizada para reduzir seus efeitos (QUADRO I) (TORRES, 2014) 
QUADRO r: Principais agentes tóxicos, doses, sintomas e antídotos utilizados na reabilitação do paciente intoxicado

\begin{tabular}{|c|c|c|c|}
\hline AGENTE TÓXICO & $\begin{array}{l}\text { DOSE } \\
\text { TÓXICA }\end{array}$ & SINTOMAS & ANTÍDOTO \\
\hline $\begin{array}{l}\text { ANTIDEPRESSIVOS } \\
\text { TRICÍCLICOS } \\
\text { (AMITRIPTILINA, } \\
\text { DOXEPINA, } \\
\text { IMIPRAMINA E } \\
\text { NORTRIPTILINA). }\end{array}$ & Io a $20 \mathrm{mg} / \mathrm{kg}$ & $\begin{array}{l}\text { Midriase, rubor } \\
\text { facial, hipertermia, } \\
\text { retenção urinária, } \\
\text { diminuição } \\
\text { motilidade intestinal, } \\
\text { ataxia, prostração, } \\
\text { alucinações, } \\
\text { convulsões, coma, } \\
\text { rigidez muscular, } \\
\text { taquicardia, } \\
\text { disritmias } \\
\text { ventriculares, } \\
\text { bloqueios do ramo, } \\
\text { alargamento QRS, } \\
\text { aumento do QT, } \\
\text { depressão } \\
\text { respiratória. }\end{array}$ & $\begin{array}{l}\text { Lavagem gástrica e } \\
\text { carvão ativado, } \\
\text { bicabornato de sódio } \\
\text { para manutenção de } \\
\mathrm{Ph} \text { sanguíneo entre } \\
7.45 \text { e } 7.55\end{array}$ \\
\hline OPIÁCEOS & - & $\begin{array}{l}\text { Miose, depressão } \\
\text { respiratória, } \\
\text { depressão } \\
\text { coma }\end{array}$ & $\begin{array}{l}\text { Naloxona IM/EV, } \\
\text { até reverter o quadro } \\
\text { (max. 2,o mg, SC, } \\
\text { IM, endotraqueal, } \\
\text { intranasal). }\end{array}$ \\
\hline ANFETAMINAS & & $\begin{array}{l}\text { Estimulantes do } \\
\text { SNC. Taquicardia, } \\
\text { hipertensão, } \\
\text { palpitações, dor } \\
\text { torácica, disritmias, } \\
\text { hipertermia, } \\
\text { sudorese, } \\
\text { desidratação, } \\
\text { agitação, } \\
\text { comportamento } \\
\text { psicótico, convulsões, } \\
\text { coma, rabdomiólise, } \\
\text { insuficiência renal. }\end{array}$ & $\begin{array}{l}\text { Lavagem gástrica e } \\
\text { carvão ativado se } \\
\text { indicado. } \\
\text { dos Redução } \\
\text { externos } \\
\text { calmo), ambímulos } \\
\text { convulsões: } \\
\text { 968diazepante }\end{array}$ \\
\hline Inibidores & $\begin{array}{l}\text { Variáveis } \\
\text { dependendo da }\end{array}$ & $\begin{array}{l}\text { Muscarínicos: } \\
\text { cialorreia, lacrimejo, } \\
\text { sudorese, } \\
\text { broncorreia, } \\
\text { bradicardia e miose. } \\
\text { Nicotínicos: }\end{array}$ & $\begin{array}{l}\text { I) Segurança de toda } \\
\text { a equipa de saúde } \\
\text { (lavagem com água e } \\
\text { sabão para evitar } \\
\text { exposição); ABCD } \\
\text { de reanimação; } 3 \text { ) Se }\end{array}$ \\
\hline
\end{tabular}




\begin{tabular}{|c|c|c|c|}
\hline colinesterases & $\begin{array}{l}\text { substância em } \\
\text { causa. }\end{array}$ & $\begin{array}{l}\text { Fasciculações, } \\
\text { taquicardia e } \\
\text { midríase. Após } \\
\text { exposição a } \\
\text { organofosforados, } \\
\text { passados dias ou } \\
\text { semanas pode surgir } \\
\text { neuropatia periférica. }\end{array}$ & $\begin{array}{l}\text { convulsões } \\
\text { (Diazepam); } \\
\text { Lavagem gástrica } \\
\text { com carvão ativado; } \\
\text { Atropina: dose } \\
\text { inicial: } 2 \mathrm{mg} \text { ( } 6 \mathrm{mg} \text { se } \\
\text { risco de vida) EV; }\end{array}$ \\
\hline Cocaína & - & $\begin{array}{l}\text { Convulsões, } \\
\text { hipertermia, } \\
\text { rabdomiólise, } \\
\text { insuficiência renal, } \\
\text { nos casos mais graves } \\
\text { disritmias e colapso } \\
\text { cardiovascular. }\end{array}$ & $\begin{array}{l}\text { Diazepam. Soros, } \\
\text { monitorização ECG, } \\
\text { suporte ventilatório } \\
\text { conforme a clínica. }\end{array}$ \\
\hline
\end{tabular}

FONTE: ADAPTADO DE TORRES, 2014

\subsection{Atuação farmacêutica na prevenção a intoxicação medicamentosa}

De acordo com a OMS mais de 50\% dos medicamentos são prescritos dispensados e vendidos de forma incorreta, pacientes também alteram por conta própria a farmacoterapia prescrita, causando mais riscos do que benefícios. A atenção farmacêutica tem como fundamento, o uso racional de medicamentos, objetivando a indicação apropriada, tempo do tratamento, redução dos efeitos adversos, interações medicamentosas e consequentemente diminuindo os riscos de intoxicação medicamentosa (OLIVEIRA et al., 2007; SOUSA, 2012).

O profissional farmacêutico tem propriedade quando o tema é o uso racional de medicamentos, pois é detentor de conhecimento técnico e de competências indispensáveis na identificação de possíveis eventos adversos ocasionados pelo uso dos medicamentos. A atuação do farmacêutico junto à sociedade é de extrema importância, assim como sua atuação integrando equipes multidisciplinares, em decorrência disso agrega valor e traz resultados positivos que beneficiam os pacientes (SANTANA, 2017).

O farmacêutico mediante a utilização da atenção farmacêutica, é um importante coadjuvante na reversão das situações de intoxicação medicamentosa, de modo que auxilia a reduzir os efeitos danosos da ingestão indevida dos medicamentos, por meio de uma farmacoterapia humanizada e individualizada, na qual se objetiva a melhoria da qualidade de vida e a restauração do estado de saúde do paciente (CALDERARI, 2017). 
Destaca-se também a atuação farmacêutica na farmacovigilância, que tem por finalidade reduzir taxas de morbimortalidade atribuídas ao uso de medicamentos, mediante a deteç̧ão prévia de possíveis problemas que os fármacos possam causar nos usuários, aperfeiçoando o uso racional de medicamentos pelos profissionais de saúde. Nesse âmbito, ante a gama de atribuições do farmacêutico, sua importância frente a vigilância de medicamentos é substancial frente a classe de profissionais a qual pertence, de modo que através do seu conhecimento técnico possa instituir consensos e auxiliar na tomada de decisões, visando aprimorar sua atuação (SANTOS et al., 202ı).

Uma das formas que o governo utiliza para diminuir o uso indiscriminado de medicamentos é com a elaboração de leis, portarias e programas educacionais de saúde, em 2007, o Ministério da Saúde criou a portaria nº 1.555 que institui o Comitê Nacional para a Promoção do Uso Racional de Medicamento, buscando o uso seguro e eficaz dos fármacos (MINISTÉRIO DA SAÚDE, 2007).

As notificações feitas ao Sistema Nacional de Vigilância Sanitária (SNVS) servem para auxiliar no reconhecimento de reações adversas ou efeitos indesejados dos remédios, melhorando o conhecimento sobre os efeitos. Baseado na Lei no 13.021/14, é obrigação do farmacêutico, no exercício de suas atividades, a notificação aos profissionais de saúde, órgãos de vigilância sanitária e laboratório responsável a respeito de intoxicações, voluntárias ou provocadas, efeitos colaterais; reações adversas e farmacodependência presenciados no decorrer de suas atividades (BRASIL, 2015).

Os medicamentos são essenciais na terapêutica de assistência de recuperação e preservação à saúde das pessoas. Porém, se utilizados de forma inadequada podem causar muitas reações, intensificando problemas de saúde e aumentando os custos do sistema de saúde. Portando o do uso racional de medicamentos orientada pelo profissional farmacêutico tem o intuito de minimizar os efeitos da tomada irregular das drogas. Desta forma compreende-se que o farmacêutico contribui de forma significativa, pois é especialista no assunto, e sua atuação integrando equipes multidisciplinares aperfeiçoar os serviços de saúde (VIEIRA, 2007). 


\section{CONCLUSÃO}

A automedicação, o uso indevido dos medicamentos e a publicidade irregular são fatores que predispõem os pacientes as intoxicações medicamentosas, e um dos desafios do profissional farmacêutico é viabilizar o uso racional dos medicamentos, desmistificar costumes e hábitos sociais que prejudicam o indivíduo.

Diante do exposto fica evidente a importância de modificações dos hábitos da sociedade em relação a exposição desnecessária, a ingestão de dosagens acima das prescritas e a tomada irregular de vários medicamentos simultaneamente, a fim de que sejam evitadas internações por intoxicação medicamentosa.

Confirmou-se nesse estudo a importância do farmacêutico como profissional mais qualificado e apto a integrar equipes multidisciplinares, pois é conhecedor técnico sobre o assunto, desta forma sua atuação tem o intuito de reparar e reduzir os danos causados pelas intoxicações medicamentosas.

\section{REFERÊNCIAS}

AQUINO, DS. Por que o uso racional de medicamentos deve ser uma prioridade? Ciência \& Saúde Coletiva, v. 13, p. 733-736, 2008.

ARRAIS, P.D., et al. Prevalence of self-medication in Brazil and associated factors. Revista de Saúde Pública, 2016, v. 50, p. 13

BARTLETT, D. Drug-Induce serotonin syndrome. Critical Care Nurse, 2017; v. 37, n. I, p. 49-54.

BERNARDES, S. S.; TURINI, C. A.; MATSUO, T. Perfil das tentativas de suicídio por sobre dose intencional de medicamentos atendidas por um Centro de Controle de Intoxicações do Paraná, Brasil. Cadernos de Saúde Pública, v. 26, n. 7, p.1366-1372, 2010 BEZERRA, A. C. P., et al. Utilização da lavagem gástrica e do carvão ativado como medidas de intervenção terapêutica na intoxicação exógena. Revista Eletrônica Acervo Saúde, 2020; v. I2, n. 12, p. 4990.

BITENCOURT NKS, BORGES LM, ALVES SMF, SOUZA FHHV. Intoxicações medicamentosas registradas pelo Centro de Informações Toxicológicas de Goiás, 2008. p. I-6. 
BRASIL. Conselho Regional de Farmácia do Estado de São Paulo. Manual de Orientação ao Farmacêutico: Lei no 13.021/2014 e Valorização Profissional / Conselho Regional de Farmácia do Estado de São Paulo. Grupo Farmácia Estabelecimento de Saúde. São Paulo, 2015 .

BRASIL. Ministério da Saúde (MS). Política nacional de medicamentos, 2002.

BRASIL. Ministério da Saúde (MS). Uso racional de medicamentos: temas selecionados Brasília: MS; 2012. (Série A. Normas e Manuais Técnicos).

CALDERARI, W. J. U. Intoxicação Medicamentosa: A Atuação do Farmacêutico. Faculdade de Educação e Meio Ambiente. Ariquemes - RO, 2017

CASTILHO, M. M., et al. A true dialytic urgency: lithium intoxication. Revista Colombiana de Nefrología; v. 5, n. 2, p. 190-194, 2018.

CRUZ, M.J.B, et al. Medication use among children o-I4 years old: population baseline study. Jornal de pediatria; v. 90, n. 6, p. 608-615, 2014.

DHAMER T, DAL-MOLIN AP, HELFER A, POSSUELO LG, CARNEIRO M, KAUFFMANN C, et al. A automedicação em acadêmicos de cursos de graduação da área da saúde em uma universidade privada do estado do Rio Grande do Sul. Rev. de Epidemiologia e Controle de Infecção; v. 2, n. 4, p. 1-3, 2012.

ESHER, A.; COUTINHO, T. Uso racional de medicamentos, farmaceuticalização e usos do metilfenidato. Ciência \& Saúde Coletiva, v. 22, p. 2571-2580, 2017.

FERRANTI, S., et al. Antiepileptic drugs: Role in pediatric poisoning. Journal of Pediatrics and Child Health, 2018; v. 54, n. 5, p. 475-479

GANDOLFI, A. MGG. Eventos toxicológicos relacionados a medicamentos no Estado de São Paulo. Rev Saúde Pública. 2006, v. 40, n. 6, p. 1056-64

GONÇALVES, C. A.; SANTOS, V. A. DOS; SARTURI, L.; GONÇALVES, C. dos S. Epidemiologia das intoxicações por medicamentos em idosos entre 2010-2018. 32f. Universidade Estadual da Paraíba, Campina Grande, 2019

GONÇALVES, Claudiana Aguilar; GONÇALVES, Cleide Avilar; SANTOS, Valdeir Areia dos; Intoxicação Medicamentosa: Relacionada Ao Uso Indiscriminado De Medicamentos. Revista Científica da Faculdade de Educação e Meio Ambiente, v. 34 8, n. I, p. I35-I43, jan.-jun., 2017 
ICTQ. Instituto de Pesquisa e Pós-Graduação do Mercado Farmacêutico. Pesquisa sobre o Uso Racional de Medicamentos no Brasil. Disponível em http://www.ictq.com.br/component/tags/tag/r9-farmaceutica. Acesso em: o7 de outubro de 202I.

KOVACS, F.T; BRITO M. F. M. Percepção da doença e automedicação em pacientes com escabiose. An Bras Dermatol, v. 8I, n. 4, p. 335-40. 2006

LIMA GB, ARAUJO EJF, SOUSA KM, BENVIDO RF, SILVA WCS, JR RACC, et al. Avaliação da utilização de medicamentos armazenados em domicílios por uma população atendida pelo PSF. Rev. Bras. Farm. 2008; v. 89, n. 2, p. I-4.

MAGALHÃES, Andrea Franco Amoras. As intoxicações no Brasil, qual a realidade? In: Laboratório De Toxicologia. Universidade de Brasília. 2016. Disponível em: https://www.toxicologia.unb.br/?pg=desc-noticias_foco\&id=36 Acesso em: o7 de outubro de 2021.

MAIOR, M. C. L. S.; OLIVEIRA, N. V. B. V. Intoxicação medicamentosa infantil: um estudo das causas e ações preventivas possíveis. Rev. Bras. Farm. Rio de Janeiro, v. 4, n. 93, p.422-430, 2012.

MARTINS, L. S.; FILHO, A. A. Uso de solução lipídica em intoxicação por betabloqueador: relato de caso. Revista Médica de Minas Gerais, 2017.

MINISTÉRIO DA SAÚDE (BR). Portaria no $\mathbf{1 . 5 5 5}$, de 27 de junho de 2007. Institui o Comitê Nacional para a Promoção do Uso Racional de Medicamentos. Brasília (DF) Diário Oficial da união. 2007

MOTA, D. M, et al. Perfil da mortalidade por intoxicação com medicamentos no Brasil, 1996-2005: retrato de uma década. 2012

NOGUEIRA, Luciano José, et al. Histórico da Evolução da Química Medicinal e a Importância da Lipofilia: de Hipócrates e Galeno a Paracelsus e as Contribuições de Overton e de Hansch. Rev. Virtual Quim. 2009

OGA, S.; CAMARGO, M. M. A.; BAtistuzZO, J. A. O. Fundamentos de Toxicologia. (4a․ ed.). São Paulo. Atheneu Editora; 2014.

OLIVEIRA MA, BERMUDEZ JAZ; OSORIO DC, SERPA CG. Assistência farmacêutica e acesso a medicamentos. Rio de Janeiro; Edi. FIOCRUZ; 2007 
OLIVEIRA, Djanane Ramalho de. Atenção Farmacêutica como Construção da Realidade. Revista Racine, v. I09 - Março/Abril de 2009.

OLSON, K. et al. Manual de toxicologia clínica. 6 ed. Porto Alegre: Artmed, 2013 PAPINI, S.; NAKAGAWA, L.E. Current status of rodenticide intoxication in Brazil: a preliminary survey from 2009 to 2011. Brazilian Archives of Biology and Technology, 2014, v. 57, n. 5 , p. $685-688$

PÉREZ, M. M. et al. Intoxicación por barbitúricos, una mirada toxicológica. Horizonte sanitario, 2019; v. I8, n. 2, p. III-II8

PONS, E.D.S., et al. Predisposing factors to the practice of self-medication in Brazil: Results from the National Survey on Access, Use and Promotion of Rational Use of Medicines (PNAUM). PLOS ONE, 2017, v. I2, n. I2, p. I-I2

SANTANA, K. S. O Papel do Profissional Farmacêutico na Promoção da Saúde E do Uso Racional de Medicamentos. Faculdade de Educação e Meio Ambiente. Ariquemes - RO, 2017

SANTOS, M. G.; CARREIRA, C. F. S.; FARIAS, S. R. S.; CANAVIEIRAS, S. A. A importância do profissional farmacêutico na farmacovigilância. Centro de Ciências da Saúde/Departamento de Ciências Farmacêuticas. 2021 Disponível em: http://www.prac.ufpb.br/anais/xenex_xienid/xi_enid/monitoriapet/RESUMOS/Area6/ 6CCSDCFPETo3-P.pdf Acesso em: o7 de outubro de 202I.

SÃO PAULO. Secretaria Municipal de Saúde. Manual de Toxicologia Clínica: Orientações para assistência e vigilância das intoxicações agudas. 2017. Disponível em: http://www.cvs.saude.sp.gov.br/up/MANUAL\%2oDE\%20TOXICOLOGIA\%20CL\%C 3 \%8DNICA\%20-\%20COVISA\%202017.pdf Acesso em: o8 de outubro de 2021.

SCHENKEL, Eloir Paulo et al. Assistência Farmacêutica: Saúde no Brasil - Contribuições para a Agenda de Prioridades de Pesquisa/Ministério da Saúde. - Brasília: Ministério da Saúde, 2004.

SECRETARIAS DE ESTADO DA SAÚDE. Sistema Estadual De Toxicovigilância. Manual de toxicovigilância. São Paulo - SP. v. I, n. 2, 2014. Disponível em: http://www.cvs.saude.sp.gov.br/up/Caderno\%2od e\%20Toxicovigil\%C3\%A2ncia\%20I\%2015.04.2014\%2ofinal.pdf. Acesso em: o8 de outubro de 2021. 
SERENO, V. M. B.; SILVA, A. S.; SILVA, G. C. da. Perfil epidemiológico das intoxicações por medicamentos no Brasil entre os anos de 2013 a 2017. Braz. J. of Develop., Curitiba, v. 6, n. 6, p.33892-33903. 2020

SILVA, V. T.; COELHO, L. M. M; SANTOS, D. B; MARTINS, L. S; SANTOS, G. B. Intoxicação por medicamentos: uma revisão de literatura com abordagem no tratamento Universidade José do Rosário Vellano (UNIFENAS), Revista Eletrônica Acervo Científico, 2021.

SINITOX. Sistema de Informações Tóxico-Farmacológicas. Registros de Intoxicações. 2012.

http://www.fiocruz.br/sinitox_novo/cgi/cgilua.exe/sys/start.htm?sid=356. Acesso em: o8 de outubro de 202I.

SKOLNIK, A.; MONAS, J. The Crashing Toxicology. Emerg Med Clin N Am, 2020; v. 38 , p. $84 \mathrm{I}-856$.

SOUSA De APR. Prescrição por denominação comum internacional: uma imposição da política do medicamento? Faculdade de Medicina da Universidade do Porto; 2012.

SOUSA HWO, SILVA JL, NETO MS. A importância do profissional farmacêutico no combate à automedicação no Brasil. Rev. Elet. Farm (ISSN ı8o8-o804). 2008; v. 5, n. I, p. I6.

SOUSA, Q. H. L. Intoxicações Medicamentosas em Crianças e Adolescentes. XI Mostra Interdisciplinar Do Curso De Enfermagem. Disponível em: http://reservas.fcrs.edu.br/index.php/eedic/article/view/4195 Acesso em: o8 de outubro de 202I.

VARGAS, D. A. Atenção Farmacêutica nas Intoxicações Medicamentosas. Faculdade de Educação e Meio Ambiente. Ariquemes-RO, 2019.

VIEIRA, Fabiola Sulpino. Possibilidades de contribuição do farmacêutico para a promoção da saúde. Cienc. Saúde coletiva. v. I2, n. I; 2007 